\title{
UM JOGO PARA TREINAMENTO DE OPERADORES DE SONDAS DE PERFURAÇÃO TERRESTRE
}

\author{
J. F. S. Chagas ${ }^{1}$, F. M. Mendes Neto ${ }^{1}$, I. S. Brasil ${ }^{1}$, R. Monteiro ${ }^{1}$, M. F. Bonates ${ }^{1}$ \\ 1Universidade Federal Rural do Semiárido - Mossoró/RN - Brasil. \\ ferdinandy@ufersa.edu.br
}

Artigo submetido em dezembro/2011 e aceito em março/2012

\section{RESUMO}

No intuito de reduzir a possibilidade de acidentes e falhas no processo de perfuração de poços de petróleo, um treinamento contínuo para os trabalhadores é altamente recomendado. Neste trabalho é proposto um jogo que simula uma plataforma de perfuração. No jogo, os jogadores são expostos a várias circunstancias nas quais eles podem exercitar seu conhecimento sobre seu trabalho e aprender o que fazer em situações adversas. Técnicas de inteligência artificial serão aplicadas ao jogo no intuito de atingir essa funcionalidade. Os recursos da aplicação incluem interação instantânea entre vários jogadores em ambiente virtual 3D. Ela é uma aplicação baseada na web, que permite jogadores remotamente executá-la em diferentes plataformas.

PALAVRAS-CHAVE: jogos sérios, jogos de treinamento, sondas de perfuração.

\section{A GAME FOR TRAINING OF OPERATORS OF LAND DRILLING RIGS}

\section{ABSTRACT}

In order to reduce the chances of accidents and failures in drilling an oil well, a continuous training to the workers is highly recommended. This paper presents a game which simulates a drilling rig. In this game, the players are exposed to various circumstances in which they can exercise their knowledge about their work and learn what to do in adverse situations. Artificial Intelligence techniques will be applied to the game in order to accomplish that feature. The application will also include instant interactions among several players in a virtual 3D environment. The game is a web-based, which allows players to remotely execute it on different platforms.

KEY-WORDS: serious games, training games, drilling rigs. 


\section{UM JOGO PARA TREINAMENTO DE OPERADORES DE SONDAS DE PERFURAÇÃO TERRESTRE}

\section{INTRODUÇÃO}

Acidentes sempre estiveram presentes na história da indústria do petróleo, causando catástrofes ambientais, óbitos, lesões e outros danos. Esse fato está diretamente ligado à complexidade do sistema de uma forma geral. Segundo Perow (1999) apud (Garcia, 2003), sistemas industriais complexos sempre estão vulneráveis a falhas, sendo, portanto, impossível eliminar os riscos inerentes a estes sistemas. Em vista disso, existe a necessidade das empresas da área petrolífera capacitarem melhor seus funcionários, promovendo treinamentos contínuos que envolvam não apenas conceitos técnicos relacionados à operação da plataforma, mas também os fatores relacionados ao trabalho em equipe e como lidar com situações adversas. A elaboração de um programa de treinamento adequado enfrenta como entraves a distribuição geográfica de funcionários e suas disponibilidades de horários. Além disso, os riscos inerentes a esta atividade acarreta em um treinamento prático de elevado custo (Thomas, 2004).

Buscando solucionar esses problemas, é proposto um jogo de computador para complementar os treinamentos convencionais. O jogo simula a operação de uma sonda de perfuração terrestre, no qual o treinando é submetido a diversas situações, diante das quais possa exercitar o conhecimento sobre seu trabalho na equipe e aprender o que fazer em condições adversas. Estudos indicam que jogadores aumentam sua concentração, seus reflexos e aprendem a lidar com situações que necessitam de tomadas de decisões rápidas (Morsi \& Jackson, 2007). Podemos observar que esses fatores são muito importantes para o treinamento de operários de uma plataforma de perfuração, devido ao complexo trabalho desempenhado por eles.

Neste trabalho é apresentada uma parte do jogo proposto, onde foi implementada a operação de conexão, que será detalhada posteriormente, e a situação de um $k^{\prime} k^{1}{ }^{1}$. Essa etapa inicial contempla um perfil de operário, dois agentes inteligentes e uma operação básica. O desenvolvimento da proposta apresentada faz parte do projeto APLMIN-RN Fortalecimento da Estrutura de Apoio à Pesquisa para o Arranjo Produtivo Mineral do Rio Grande do Norte. Um projeto do CNPq com apoio da Petrobras, que acompanha o desenvolvimento e fornece informações e materiais úteis sobre os processos de produção de petróleo.

\section{APRENDIZAGEM COLABORATIVA A DISTÂNCIA BASEADA EM JOGOS}

$\mathrm{Na}$ educação, o que se tem visto são novas metodologias e abordagens educacionais sendo criadas e recriadas com o intuito de alcançar melhores resultados com um público cada vez mais dinâmico e diversificado. Uma dessas abordagens é o uso de jogos eletrônicos no contexto educacional, que segundo SGI - Serious Games Initiative - em 2010, vem sendo globalmente discutido e aplicado na educação formal e não-formal.

Para Gadotti (2005), educação formal representa um conjunto de atividades educacionais com objetivos claros e bem definidos, que obedecem a diretrizes educacionais centralizadas e são executadas nos limites das escolas e universidades. Já a educação não-

\footnotetext{
${ }^{1}$ Fluxo indesejável do fluido contido numa formação geológica para dentro do poço HOLOS, Ano 28, Vol 1
} 
formal é mais difusa, menos hierárquica, desburocratizada e normalmente é executada fora dos limites do sistema formal das escolas e universidades.

Nos diversos níveis educacionais, um dos fatores que mais preocupa o professor é o nível motivacional de seus alunos. Em (Sandford et al., 2006), observou-se que 53\% dos professores encaram a motivação de seus alunos como razão primária para se utilizar jogos educacionais. Contudo, os autores defendem que o uso de jogos em ambientes educacionais não garante motivação por si só. É preciso atentar para as características dos jogos que melhor incentivam o compromisso dos estudantes.

No contexto da Engenharia de Software, inúmeras iniciativas surgiram com o objetivo de se utilizar das qualidades lúdicas e motivadoras que os jogos possuem, no ensino ou treinamento de novos profissionais, de modo a suprir as limitações impostas pelo tempo e/ou formato das disciplinas e cursos formais dessa área. Em (Fernandes \& Werner, 2009), é analisada e discutida a viabilidade do uso de jogos com essa finalidade. Nesse trabalho, as autoras concluem que se trata de uma abordagem bastante promissora e aconselham que os próximos jogos na área possuam características baseadas em critérios bem definidos, cuja aplicabilidade dos resultados possa ser mensurada.

\section{A OPERAÇÃO DE PERFURAÇÃO DE POÇOS DE PETRÓLEO TERRESTRES}

Durante a operação de perfuração existem diversos procedimentos que são realizados pelos operários como: manobra, conexão, tratamento do fluido de perfuração e pescagem. Todos esses procedimentos e demais operações são realizadas pelos operários a partir de uma plataforma de perfuração semelhante à ilustrada na Figura 1-a.

A perfuração de um poço de petróleo é realizada através da quebra de rochas utilizando uma sonda de perfuração, que é uma estrutura desenvolvida com as especificações técnicas necessárias para dar suporte à operação. Na sonda, operários trabalham executando os processos de operação da coluna de perfuração, estrutura que compõe a sonda. A coluna de perfuração é um composto de segmentos tubulares, que irão conduzir a broca, um componente que quebrará a rocha abaixo do solo até chegar às rochas onde está depositado o petróleo.

A operação de perfuração é realizada em diversas fases. Cada fase pode ser identificada pelo diâmetro dos segmentos utilizados. A cada etapa, uma camada de revestimento metálico é introduzida na parede do poço para dar segurança à operação. Cada camada possui um diâmetro diferente, do maior para o menor, assim como os segmentos. À medida que a perfuração vai avançando, são adicionados segmentos tubulares para prolongar a coluna de perfuração. Desta forma, prolonga-se o alcance da broca até atingir o objetivo, que são as rochas que guardam o petróleo. O processo pode ser melhor compreendido por meio da Figura 1-b. 


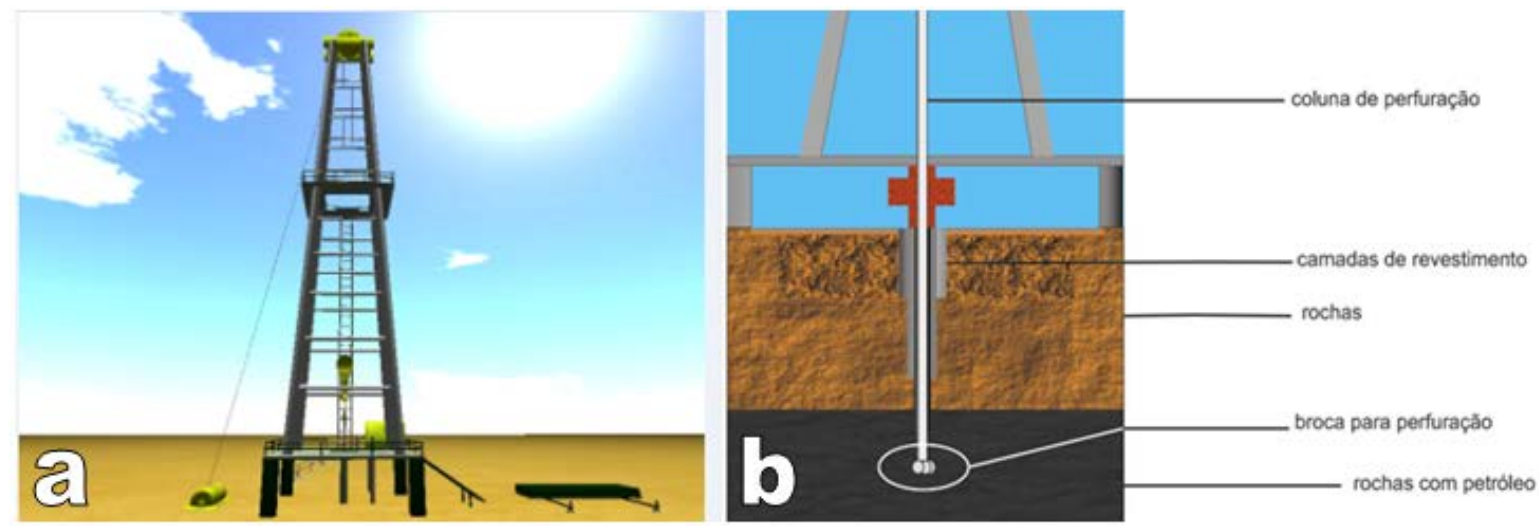

Figura 1 - Plataforma de perfuração carregada no jogo (a). Detalhamento da operação (b)

\section{VISÃO GERAL DO JOGO}

Direcionado para um ambiente multijogador, o jogo utilizará técnicas de inteligência artificial com o intuito de gerar desafios semelhantes aos enfrentados pelos profissionais que trabalham na operação de perfuração de poços terrestres. Para isso, o jogo simula o trabalho de uma equipe de perfuração, onde os jogadores formarão a equipe. Na equipe, cada um dos treinandos ocupará um cargo específico relativo às operações da sonda de perfuração (por exemplo, auxiliar de plataformista, plataformista, torrista, operador de sonda, encarregado de sonda, engenheiro mecânico, etc.) e, caso a equipe não esteja completa, o sistema incluirá agentes inteligentes programados com os perfis dos cargos necessários para completar a equipe. Os agentes, com o intuito de simular situações reais de trabalho, poderão executar operações de forma correta ou incorreta, gerando mais desafios para a equipe.

O mundo virtual do jogo será adaptado para gerar situações adversas baseadas em falhas, acidentes e outras circunstâncias decorrentes da operação de perfuração do poço de petróleo. Os desafios serão compatíveis com os níveis dos jogadores, variando a quantidade e a complexidade das situações adversas para permitir essa compatibilidade.

Algumas questões requerem consideração ao se projetar um jogo multijogador para avaliar o conhecimento e habilidade dos treinandos. Para isso, o jogo será apoiado por uma teoria de aprendizagem que será utilizada para monitorar e avaliar o desempenho dos jogadores.

\section{FERRAMENTAS UTILIZADAS}

Para o desenvolvimento do jogo são utilizadas diferentes ferramentas: o Blender, que é um software de modelagem e animação 3D de código aberto (Brito, 2007); JPCT (http://www.jpct.net), um motor gráfico para desenvolvimento de aplicações gráficas em 3D para navegador; JADE (Java Agent DEvelopment Framework) (Bellifemine et al., 2007), uma plataforma para desenvolvimento e execução de sistemas multiagentes; e o NetBeans (http://www.netbeans.org), um ambiente para desenvolvimento de programas baseados na linguagem de programação Java, para integração das tecnologias envolvidas. 
Com o Blender, a partir de imagens reais dos componentes que integram a plataforma de perfuração, foram criados os modelos em 3D utilizados no jogo. Para chegar a esse resultado, foi realizado um estudo dos recursos disponíveis na ferramenta. Este estudo possibilitou obtenção dos conhecimentos necessários para buscar retratar aproximadamente a forma real dos componentes, o que é uma das características fundamentais de um simulador.

Foram utilizadas outras ferramentas, como o banco de dados MySql (MYSQL, 2012) para a persistência dos dados, jFuzzyLogic (JFUZZYLOGIC, 2012), como mecanismo de inferência da lógica fuzzy, e H-N2N (BURLAMAQUI, 2006) para suporte ao ambiente multijogador.

\section{AGENTE E LÓGICA FUZZY}

Segundo Wooldridge (2002), um agente é um sistema computacional que está situado em algum ambiente e que é capaz de efetuar ações autônomas neste ambiente a fim de cumprir os objetivos para os quais ele foi feito.

Para que os agentes não executem ações sempre "certas", como seria o caso de um agente apenas baseado em regras do tipo "condição-ação", pode-se incluir um certo grau de incerteza na tomada de decisão dos agentes usando a Lógica Fuzzy (FL), ou Nebulosa (ZADEH, 1965). A FL propõe que no lugar de valores únicos sejam usados vários valores no intervalo de zero até um, representando uma variação entre o completo falso e a verdade absoluta. Um exemplo prático para entendimento é quando é medida a temperatura de algo, no caso, utilizando os extremos quente e frio, no entanto, pode-se admitir também os estados intermediários, como morno.

O funcionamento do mecanismo de inferência Fuzzy é representado pela Figura 2. A primeira etapa é a definição das variáveis de entrada e saída e suas respectivas funções de inferência. Em seguida, inicia-se o processo de fuzzificação, que transforma os valores reais de entrada (no exemplo da Figura 2, 50 e 100) em valores linguísticos (na figura, baixo e alto) a partir da sua função de inferência especifica $(f(x)$, sendo $x$ a variável). Na terceira etapa, o mecanismo de inferência deduz a variável de saída a partir das entradas linguísticas e das regras de inferência definidas por um especialista. $O$ último passo consiste em traduzir o valor linguístico de saída (na figura, médio) para valor real (na figura, estado) a partir de um algoritmo de defuzzificação que consulta as funções de inferência. 


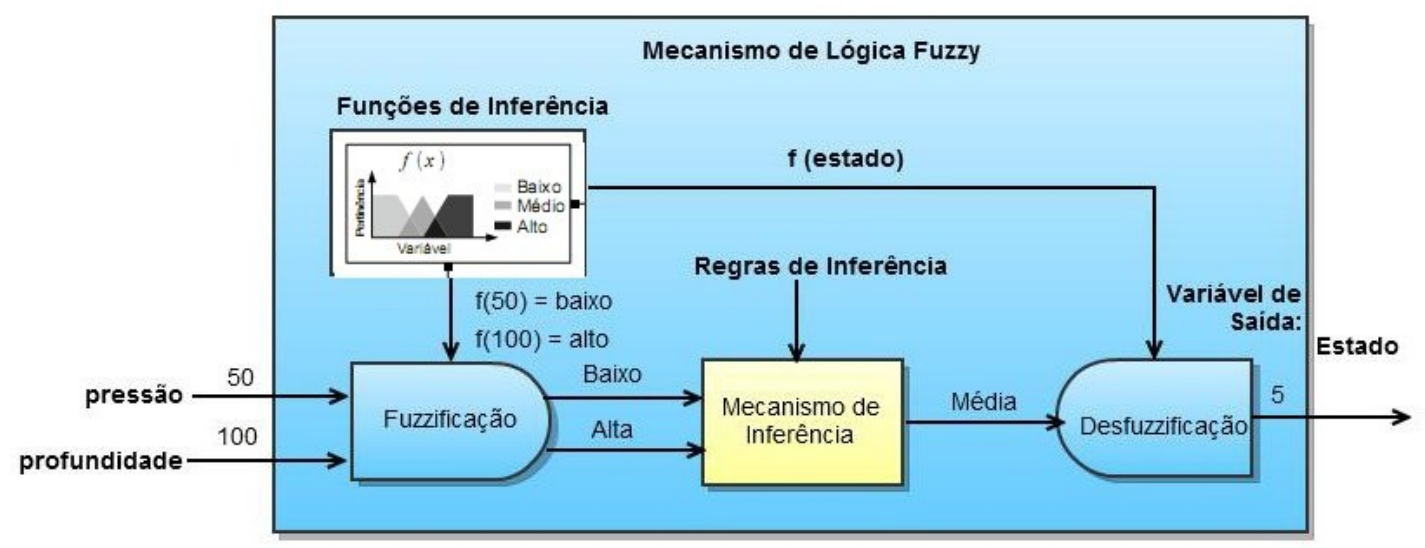

Figura 2 -- Funcionamento da representação Fuzzy

\section{A IMPLEMENTAÇÃO}

O mundo do jogo é baseado no ambiente da operação de perfuração de poços terrestres. Foram modelados o mundo, a plataforma, um avatar e algumas ferramentas utilizadas pelos operários na execução de suas atividades na sonda de perfuração. A Figura 3-a mostra a tela do jogo.

A equipe de perfuração é composta por diferentes profissionais. O jogo de treinamento é direcionado a três tipos de profissionais: o plataformista, o torrista e o operador de sonda. Inicialmente foi escolhido para desenvolvimento o perfil do plataformista, que é o membro da equipe de perfuração responsável por: conectar os tubos à coluna de perfuração e sondagem; conectar brocas na coluna, posicionando-as na mesa rotativa; deixar a coluna pronta para as operações de sondagem; lubrificar e abastecer componentes de guincho e motores durante as operações de sondagem; auxiliar o torrista nas tarefas de adicionamento de elementos químicos para o tratamento da lama, entre outras tarefas.

O objetivo do jogador, no estado atual do jogo, é realizar a operação de conexão, que é o procedimento de adicionar novos segmentos à coluna de perfuração. Para realizar essa tarefa, o jogador precisa se dirigir à plataforma e acionar a ferramenta cunha, que é uma ferramenta utilizada para segurar, sempre que for preciso, a coluna de perfuração. $O$ jogador deve acionar a ferramenta e posicioná-la corretamente utilizando o mouse. Após posicionar a cunha, a coluna é liberada pelo elevador e o guincho será posicionado para adição de um novo segmento. Como o guincho não é controlado pelo jogador, ele deve seguir o procedimento de conexão sem controlá-lo. Através do painel de controle do jogador (ver Figura 3-b), um novo segmento é adicionado ao guincho no elevador, que desce em direção à coluna, onde deverá ser realizada a operação de conexão. 

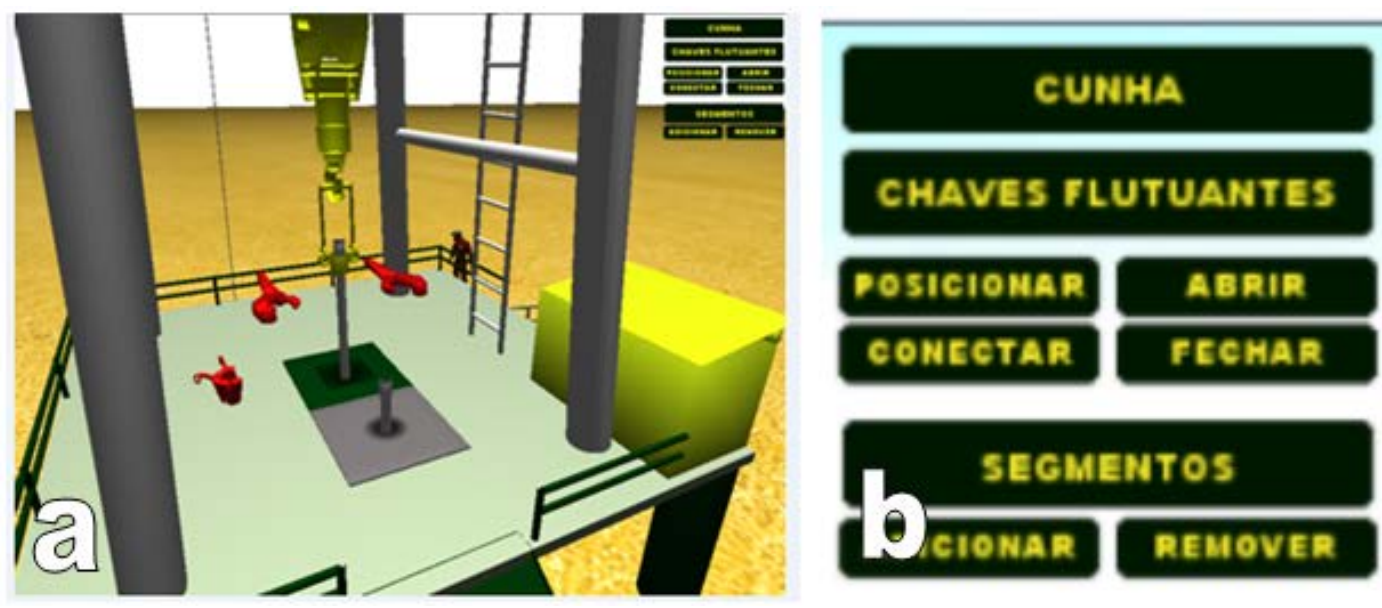

Figura 3 - Visão do jogador (a). Painel de comandos (b).

O guincho posiciona a extremidade inferior do segmento na extremidade superior da coluna apoiada. $O$ jogador deve acionar as ferramentas chaves flutuantes para poder efetuar a conexão do novo segmento à coluna. Caso a operação seja realizada com sucesso, ao ser retirada a cunha, a coluna estará novamente segura ao guincho pelo elevador, que será posicionado novamente para uma nova conexão. Para a realização do procedimento com sucesso, o jogador deverá ter o conhecimento de utilização das ferramentas e dos procedimentos para operá-las.

Para controlar o guincho utilizado na conexão, é utilizado um agente reativo simples. Este agente possui um plano de execução. A operação é iniciada após o agente detectar que o jogador (plataformista) posicionou corretamente a ferramenta cunha. Esta é uma medida de segurança que é de responsabilidade do plataformista. Em seguida, o agente libera a coluna de perfuração, eleva o guincho e aguarda que seja adicionado um novo segmento a sua extremidade. Quando o agente detecta que foi adicionado um novo elemento, ele desce o segmento até a posição correta para conexão. Como o posicionamento varia, o agente calcula a distância entre o novo segmento e a extremidade da coluna de perfuração que está apoiada pela cunha. Esse processo é realizado várias vezes durante a perfuração de um poço de petróleo.

O guincho na operação real é controlado pelo operador de sonda, que conhece o procedimento e verifica visualmente o trabalho do plataformista. No jogo proposto, um agente simula esse comportamento.

$\mathrm{Na} \mathrm{FL}$, as regras são o núcleo do sistema, pois é onde é formada a base de conhecimento usada pelo sistema de inferência fuzzy. Elas podem ser obtidas através de conhecimento especialista ou extraídas de dados numéricos e são expressas na forma de estruturas SE-ENTÃO.

O sistema fuzzy que controla o Agente Poço é formado, basicamente, por três variáveis de entrada e duas de saída. As variáveis de entrada são "pressão da formação", "pressão da coluna" e "profundidade", as duas primeiras contendo quatro conjuntos de valores (baixa, media, alta, muito alta) e a terceira contendo três conjuntos (pequena, média, grande). As 
variáveis de saída são "kick" e "desgaste da broca", contendo a primeira variável quatro conjuntos de valores (nulo, baixo, médio, alto), e a segunda os conjuntos (nenhum, baixo, médio, alto).

Foi utilizado um conjunto que variou de 10 a 15 regras para descrever o comportamento do Agente Poço. Algumas das regras que foram usadas para gerar situações adversas são dadas a seguir:

- Se pressão da coluna é muito alta e pressão da formação é baixa então kick é nulo;

- Se pressão da coluna é alta e pressão da formação é muito alta então kick é médio;

- Se profundidade é pequena e pressão da formação alta então desgaste da broca é médio;

- Se pressão da coluna é baixa e pressão da formação é alta então kick é alto;

- Se pressão da formação é alta e profundidade é grande então desgaste da broca é alto.

No modelo de interação entre os agentes apresentado na Figura 4, é possível visualizar as interações que ocorrem entre os agentes e os recursos que são consultados por cada um dos tipos de agentes. O diagrama mostra o comportamento no momento que o agente sondador acessa a sala de jogo até o momento que ele efetua alguma ação no ambiente. É possível perceber que toda a interação entre jogadores ocorre através do framework H-N2N.

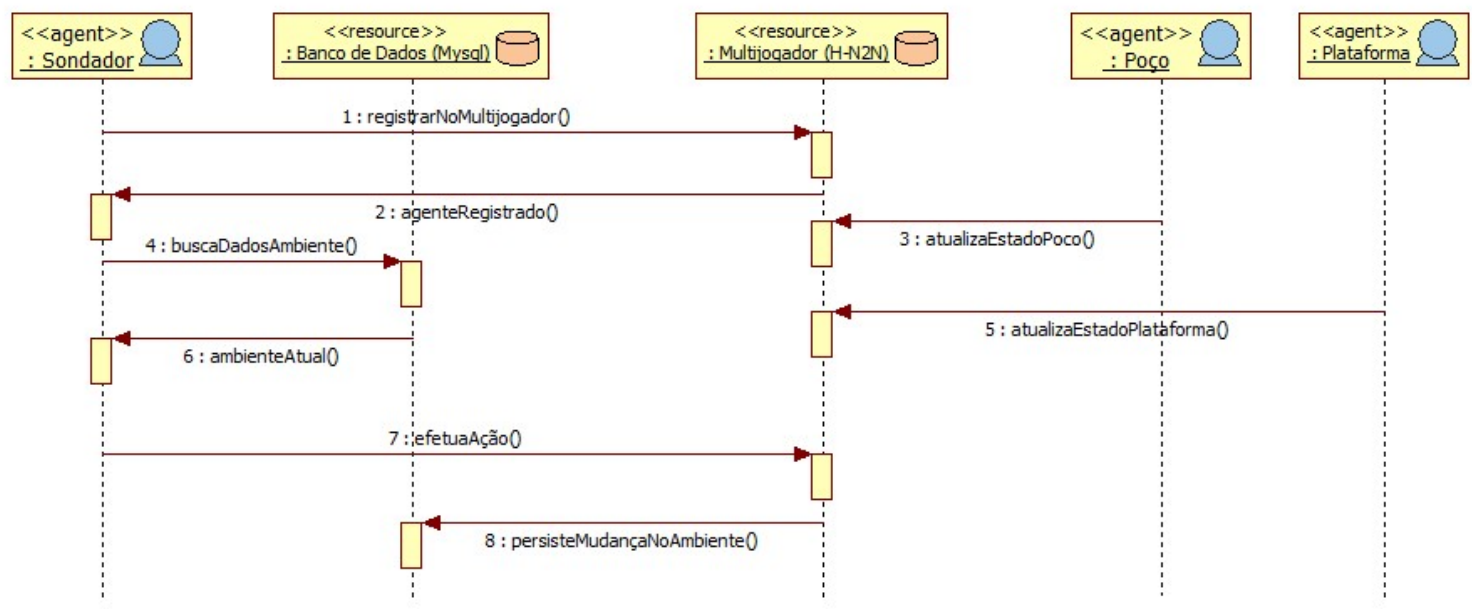

Figura 4 - Modelo de interação entre os agentes.

No modelo apresentado na Figura 4, é possível visualizar toda a interação que ocorre em um agente, nesse caso focado no agente Sondador. Entretanto, esse fluxo é similar para todos os agentes que (i) inicialmente se registram no ambiente multijogador (H-N2N); após isso, (ii) recebem uma confirmação de registro e, a partir desse momento, (iii) passam a receber todas as mensagens do ambiente. Após receber a confirmação do registro, (iv) os agentes passam a consultar o estado atual do ambiente, persistido no banco de dados. Em 
seguida, efetuam uma ação (v) enviando uma mensagem para o ambiente multijogador. Finalmente, (vi) a ação é persistida no banco de dados e enviada para os outros participantes.

Os componentes e ferramentas carregadas no jogo podem ser observadas na Figura 5.
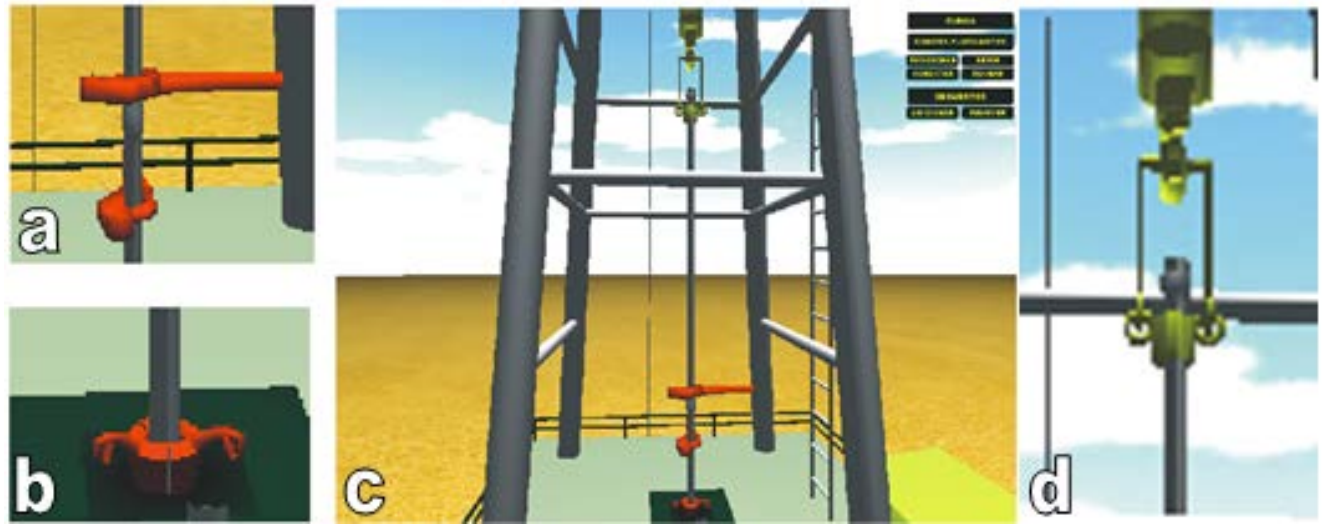

Figura 5 - Chaves flutuantes (a). Cunha(b). Visão da operação de conexão (c).

\section{CONSIDERAÇÕES FINAIS}

O jogo educacional proposto neste trabalho é um ambiente 3D didático baseado na simulação do processo de perfuração de poços de petróleo. O cenário é uma locação onde estão modelados os principais elementos do jogo. Usando o motor 3D jPCT foi criado um ambiente cliente-servidor onde o jogador pode navegar e interagir com os objetos da simulação. Foram inseridos vários elementos das atividades de perfuração, e um perfil de trabalhador foi escolhido para essa primeira etapa. A princípio, com uma etapa inicial implementada, já podemos visualizar parte dos benefícios, como a atratividade e redução dos riscos de treinamentos em ambientes reais, que a proposta do jogo sugere. Além disso, o projeto obteve uma boa receptividade da Petrobras por parte dos funcionários que acompanham diretamente o projeto.

O ambiente virtual promove a imersão do jogador, que pode facilmente aumentar a difusão do conhecimento acerca das atividades, buscando assim, com o treinamento contínuo, a mitigação de riscos à atividade de perfuração de poços de petróleo. Além dos baixos custos e redução dos riscos, vimos que podemos utilizar os jogos como uma forma atrativa de aquisição de conhecimento, respeitando o ritmo de aprendizado de cada um, visto que o treinamento faz o treinando refletir sobre seu conhecimento e rever seus conceitos.

Em trabalhos futuros, buscaremos contemplar outros perfis, outras operações em um ambiente multijogador rodando no navegador, buscando explorar as vantagens da aprendizagem colaborativa e completar outros aspectos do treinamento. Quanto ao desenvolvimento de agentes inteligentes, serão utilizadas metodologias amplamente adotadas para agregar qualidade ao projeto. 


\section{REFERÊNCIAS BIBLIOGRÁFICAS}

1. BELLIFEMINE, F., C., G., \& D., G. Developing multi-agent systems with JADE. John Wiley \& Sons Ltd, England, 2007.

2. BRITO, A. Blender 3D-Guia do Usuário. Novatec Editora, 2th edition, 2007.

3. BURLAMAQUI, A. et al. H-n2n - uma soluçãoao escalável para ambientes virtuais colaborativos massivos. XII Simpósio Brasileiro de Sistemas Multimídia e Web. [S.I.]: [s.n.]. 2006.

4. FERNANDES, L. \& WERNER, C. Sobre o uso de Jogos Digitais para o Ensino de Engenharia de Software. Simpósio Brasileiro de Engenharia de Software, 2009.

5. GARCIA, K. Análise dos sinais precursores do acidente da p-34. 2을 Congresso de Petróleo e Gás, 2003.

6. JFUZZYLOGIC. JFuzzyLogic, 2011. Disponivel em: <http://jfuzzylogic.sourceforge.net/html/index.html>. Acesso em: 10 jan. 2012.

7. MORSI, R. \& JACKSON, E. Playing and learning? educational gaming for engineering education. In Frontiers In Education Conference - Global Engineering: Knowledge Without Borders, Opportunities Without Passports, 2007. FIE '07. 37th Annual, pages F2H-1-F2H6, 2007.

8. MYSQL. MySQL: The world's most popular open source database. Site oficial do MySQL, 2011. Disponivel em: <http://www.mysql.com/>. Acesso em: 15 dez. 2011.

9. SANDFORD, R., ULICSAK, M., FACER, K., \& RUDD, T. (2006). Teaching with games: Using commercial off-the-shelf computer games in formal education. Bristol: Futurelab, 2006.

10. THOMAS, J. E. Fundamentos da Engenharia do Petróleo. Editora Inteciência, 2a edição, 2004.

11. WOOLDRIDGE, M. An Introduction to Multiagent Systems. [S.I.]: John Wiley \& Sons, Inc. New York, NY, USA, 2002.

12. ZADEH, Lofti. Fuzzy Sets. Information and Control. [s.I.]: No 8, p. 338-353, 1965. 